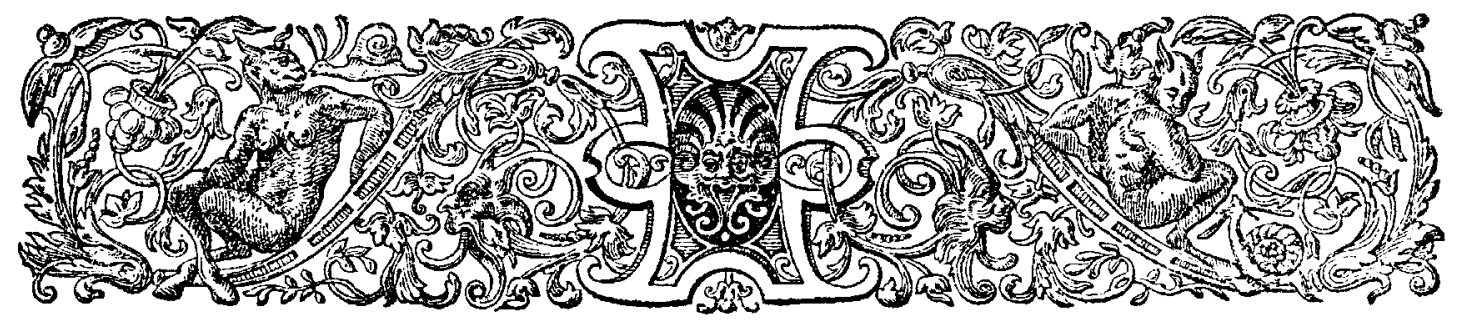

\title{
Ein altniederländisches Altarwerk in Rom von
}

\author{
Dr. HEINRICH WEIZSÄCKER.
}

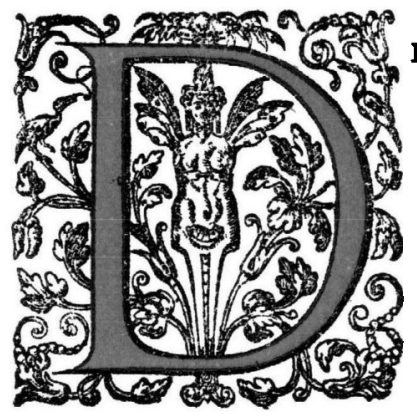

ER kleinen, hinreichend bekannten Zahl altniederländischer Altargemälde, die sich in italienischen Kirchen vortinden oder aus solchen stammen, möchte ich in den nachstehenden Zeilen ein Meisterwerk des sechzehnten Jahrhunderts hinzufügen, das in der kunstgeschichtlichen Fachliteratur bisher noch keine Erwähnung gefunden hat, und dessen Kenntnis ich selbst nur der Beschäftigung mit einigen römischen Geschichtsquellen aus späterer Zeit verdanke, die einen solchen Fund in keiner Weise vorher erwarten liessen.

Wenn man auf dem Wege, der an der Aussenseite der Peterskirche vorbei zu dem Archiv und zu den Sammlungen des Vatikans tührt die Kolonnaden des Bernini hinter sich gelassen hat, so öftnet sich links der Blick auf den sogenannten Campo Santo dei Tedeschi, die eine der beiden ehrwürdigen Stiftungen, die unter den verschiedenen nationalen kirchlichen Niederlassungen in Rom seit ältester Zeit den deutschen Namen vertreten. Über die Mauern des kleinen Friedhofes, welcher der ganzen Anlage ihren Namen gegeben hat, ragen $Z$ ypressen und die Laubkronen hoher Eukalyptusbäume hinaus ; "Teutones In Pace" lautet die schlichte Inschritt am Gittertore des Begräbnisplatzes, der bis in die jüngste Zeit seiner Bestimmung als solcher gedient hat. Mit ihm und mit dem angrenzenden Pil- 
gerhause „Zu Unser Lieben Frauen Schmerzen vom Deutschen Gottesacker" ist von Anbeginn eine Kapelle oder Kirche verbunden gewesen, deren heutiger Bau, S. Maria di Campo Santo, oder, wie sie in den älteren Romführern in der Regel heisst, Santa Maria della Pietà in Campo Santo, im letzten Viertel des $\mathbf{r} 5$ und im Beginn des I6 Jahrhunderts errichtet worden ist. 1) Den Hochaltar dieser Kirche, die, mit dem Hospiz verwachsen wie sie ist, nach aussen kaum sichtbar hervortritt, schmückte einst ein dreiteiliger gemalter Schrein von beträchtlichen Dimensionen, dessen Bestandteile an Ort und Stelle, wenn auch nicht mehr in ihrer ursprünglichen Zusammensetzung noch vorhanden sind. Diese sind es, welche den Gegenstand der nachfolgenden Mitteilungen bilden. In seiner anfänglichen Gestalt hat sich das Werk bis zum Jahre I 705 erhalten, in welchem es einem neven Tabernakel barocken Stiles weichen musste. In diesem letzten fand das Mittelst ück des bisherigen Altares aufs neue Verwendung, dagegen wurden die auf Vorderund Rückseite bemalten Flügel gespalten und die auf solche Weise gewonnenen vier Tafelbilder anderweitig untergebracht. ${ }^{2}$ ) Bei einer umfassenden Renovierung des Kircheninneren, die in den siebziger Jahren des vorigen Jahrhunderts stattfand, wurden schliesslich die sämtlichen Gemälde des ersten Hochaltares an den nach Osten und Westen gerichteten Seitenwänden des Chores aufgehängt. Was die Gegenstände anlangt (s. die Tafeln) so zeigt das einstige Mittelbild eine Darstellung, die, wie wir in Bälde sehen werden, aus den Elementen einer Beweinung und einer Grablegung Christi zusammengesetzt ist, die von uns aber, dem Namen des Gotteshauses entsprechend, doch wohl am passendsten als "Vesperbild", als Klage um den heiligen Leichnam aufzufassen sein wird. Auf den Flügeln erschienen in der alten Aufstellung als Innenbilder links die Heiligen Petrus und Johannes d. T., rechts Paulus und Jakobus der Aeltere. Bei geschlossenen Flugeln sah man eine thronende h. Anna Selbdritt auf der einen, auf der anderen Seite die Begegnung von Joachim und Anna an der Goldenen Pforte. 3) Bei der Veränderung, die mit dem Werke 1705 vorgenommen wurde, wie bei einer nachfolgenden Restaurierung ist den Malereien auf schlimme Weise mitgespielt worden. Es kam darüber sogar

1) Vergl. Anton de WaAl, der Campo Santo der Deutschen zu Rom, Freiburg, i. B. x896, S. 54. 61. ff.

2) Ebenda S. $2 \times 5$.

3) Diese Zusammenstellung ergibt sich aus der von JoHannes Antontus Brutius ( $\dagger$ r 692 ) verfassten Beschreibung der Kirchen Koms, deren Manuskript das Vatikanische Archiv bewahrt. Hicr heisst es in dem Abschnitte De Archisodalitate Diua In Campo Sancto (Jo. ANT. BRUTiI Opera T. XIV fol. 58): Ara maxima mediam sedem [ecclesiae] obtinet, Pietatis imago ornat, Virginis nimirum cuius in sinu Christus mortuus Vtrumqe latus cingunt Diuorum effigies. Ostio clauditur, quod est depictum, cum tabula armarii speciem praebeat. Ebenso in der italienischen Version des Textes (a.a. O. T. XX, fol, 74): Nel mezzo e l'altar maggiore con l'imagine della Pietà, cioè della Beatissima Vergine con Christo morto in seno, e da ambi i lati le stanno atorno altre figure de' Santi, e questo si puo chiudere. con le sue porte pur pinte, essendo il quadro in foggia d'un armario... 
zu einem Prozess mit den „colorari” die sie auszubessern ubernommen hatten, und die schliesslich mit einer Busse von hundert Scudi für den angerichteten Schaden einstehen mussten. ") Trotz dieser Unbilden, deren Spuren noch heute, namentlich an den Flügelbildern sichtbar sind, ist es doch ein ungewöhnlich bedeutender Eindruck, den die in lebensgrossen Figuren ausgeführten Gemälde nicht nur in kompositioneller Hinsicht sondern auch durch die Vorzüge der Ausführung in allen Einzeldingen gewähren. Was aber geradezu überraschend auf den von Norden kommenden Beschauer wirkt, das ist neben zahlreichen Entlehnungen aus dem italienischen Formenschatz, die sie enthalten, der ausgesprochen germanische Grundcharakter, oder sagen wir lieber gleich der spezifisch niederländische Typus, den diese Tafeln zur Schau tragen. Irgend eine Spur einer Bezeichnung tragen sie nicht. Und so verbergen sie, imponierend und rätselhaft zugleich, wie sie uns gegenübertreten, auf der einen Seite das Geheimnis ihres Ursprungs, während sie auf der anderen doch nur umso lebhafter in uns den Wunsch rege machen, die Zeit und die sonstigen, in der kunstlerischen Arbeit selbst gegebenen Voraussetzungen ihrer Entstehung tiefer zu ergründen.

Indem wir der Lösung dieser Aufgabe nähertreten, müssen wir allerdings auf eine Hoffnung von vornherin verzichten, nämlich die, als ob aus der umfänglichen Literatur der römischen Ortsbeschreibungen aus älterer oder neurer Zeit und aus den Mitteilungen, die sie über die Kunstwerke von S. Maria di Campo Santo enthalten, irgend eine Unterstützung unsrer Absicht zu gewinnen sei. Sehr wortkarg ist von vornherein die Mirabilienliteratur des 16 Jahrhunderts, aber auch die ausfuhrlicheren Kunsttopographien, die mit dem; I7 Jahrhundert einsetzen, darunter selbst so vielbenutzte Werke wie die von TItI, CecconI, VASI, NibBY, ARMELlini bis auf die neueste Publikation von DIEGo ANGELI erweisen sich für unseren $Z$ weck als nicht brauchbar. Was sie bieten sind nur kritiklose Vermutungen, die sich in deutlich erkennbarem Erbgang, aber ohne einen tatsächlichen Traditionswert, vom ersten bis zum letzten Autor fortpllanzen, und auf die hier einzugehen sich nicht verlohnt. Umso bedeutsamer ist uns dagegen eine Nachricht, die ANTON DE WAAL, der derzeitige Rektor des Hospizes, im Hinblick auf die grundlegende Frage der Entstehungszeit des Werkes in seiner oben schon von uns zitierten Geschichte des Camposanto mitteilt, die zugleich eine Geschichte der mit dieser Stiftung seit dem 15 Jahrhundert verbundenen Bruderschaft enthält. 2) Zu den wenigen Bestandteilen des Archives dieser Bruderschaft, die sich aus der Zeit vor der Plünderung Roms durch die Kaiserlichen im Jahre 1527 bis heute erhalten

1) DE WAAL a. a. O.S. 216.

2) a. a. O. S. 92 . 
haben, gehört ein Wirtschaftsbuch, dessen Eintragungen von I 50 I bis I 557 gehen. Hier findet sich zum Jahre 1502 die folgende Zahlungsnotiz, die den Choraltar betrifft: „Item soluerunt [officiales] pro tabula Beate Marie Camposancti in choro in tota summa ducatos de carlenis videlicet ducentos et quinquaginta duo et hoc scriptum pro memoria anno I 502". ') Ich verdanke der liebenswurdigen Zuvorkommenheit von Monsignore DE WAAL nächst der Erlaubnis, die in Rede stehenden Tafeln hier in Abbildung wiederzugeben, auch die Kenntnis jener Quellennachricht, von der mir in dem Archiv des Camposanto die hier mitgeteilte Abschrift zu nehmen gestattet war. Ein Ausgangspunkt für unsere Untersuchung ist ohne Zweifel mit ihr gegeben, wennschon ein solcher, den wir uns nicht ungepruff zu eigen machen dürfen. Der Name eines Künstlers ist wie man sieht auch hier nicht genannt, und es beruhrt uns nur wie einer jener neckischen Zufälle, die in der kunstgeschichtlichen Quellentorschung so oft ihre Hand im Spiele haben, wenn aut demselben Blatte der Name eines anderen Malers, Magister Jacobus, Erwähnung findet, der im Jahre vorher für dieselbe Bruderschaft über einer Pforte des Camposanto ein Gemälde der Pietà ausgeführt hat, das nicht mehr erhalten ist. ") Ebensowenig lässt unsere urkundliche Notiz von dem Gegenstande der fur den Choraltar gemalten Tafel erkennen. Ist es bei so mangelhaften äusseren Anhaltspunkten dennoch erlaubt, die hier erwähnte Lieferung auf die nachweislich von dem ehemaligen Hochaltar stammenden und noch vorhandenen Gemälde zu beziehen?

In jedem Falle steht nichts von alledem, was uns sonst über die Geschichte des Gotteshauses und seiner Innenausstattung bekannt ist, mit einer solchen Vermutung im Widerspruch. Nach DE WAAL erfolgte die Konsekration des Hochaltares gleichzeitig mit der Einweihung der neuen Kirche im Jahre I 50 I 3), jedoch geschah für die Ausstattung des Kircheninneren, abgesehen von der Beschaffung des Altaraufsatzes im darauffolgenden Jahre, nichts bis zum Jahre 1517 , in welchem acht Nebenaltäre auf einmal geweiht wurden. 4) Von 1536 , neun Jahre nach dem „Sacco di Roma", haben wir sodann ein Altarverzeichnis, das in einem Inventar der im Besitz der Bruderschaft befindlichen beweglichen Habe enthalten ist 5) und das erkennen lässt, dass die Gesamtzahl der Altäre in der $Z$ wischenzeit nicht verändert worden ist. Von einer Veränderung ist aber auch hinsichtlich des Hochaltares weder in dieser noch in der darauftolgenden Zeit bis zum Jahre 1705 die

1) Arch. Campi Sancti. Primus liber decretorum Campi Sancti de Urbe, fol. 2 v.

2) Ebenda fol, 2: Item nos officiales dedimus magistro Jacobo pinctori carlinos viginti quinque pro una Sancta Maria nuncupata pieta de super portam primam in Campo Sancto die XXX maij [1501].

3) a. a. O. S. 64 .

4) a. a. O. S. 5 I ff., 64 .

5) Arch. Campi Sancti. Liber primus scripturarum Archiconfraternitatis Beatae Mariae Campi Sancti fol 218. Oud-Holland, IgI 3 
Rede, und es ist auch wenig wahrscheinlich, dass eine solche vorgenommen worden wäre, nachdem einmal die zwar nicht übertriebene aber doch verhältnismässig stattliche Summe von 250 Karlin für dessen Retabulum unmittelbar nach der Kirchweihe ausgegeben worden war. Es ist aber auch ferner in dem Stilcharakter der uns erhaitenen Tafein nichts enthalten, was etwa der Annahme widerspräche, dass sie identisch mit den Bestandteilen jenes Altarwerkes seien, das laut Ausweis des erwähnten Rechnungsbuches im Jahre I 502 zur Ausführung gelangte, es spricht im Gegenteil gerade in stilistischer Hinsicht Manches dafür.

Ich rechne $z u$ den einer solchen Voraussetzung günstigen Momenten in erster Linie den eigentümlich quattrocentistischen Charakter, welchen an den in Frage stehenden Malereien die Mehrzahl jener Teile aufweist, die auf Einflüsse aus italienischer Schule zurückgehen. Ihr Urheber hat vor allem Perugino studiert. So sind auf der Innenseite des linken Flügels die Heiligen Petrus und Johannes Baptista in der Gesamthaltung wie namentlich in der Beinstellung und dem Gewandarrangement zwei echt perugineske Figuren. Will man dafür Analogien nennen, die auch gegenständlich übereinstimmen, so kann man etwa auf die Figuren des Petrus in dem Altarbilde van S. Maria Nuova in Fano, oder dem der Madonna mit vier Heiligen in der Wiener Galerie, des Johannes in dem Predellenbilde der Taufe Christi, das die Galerie von Rouen besitz, hinweisen. ") Ich behaupte damit selbstverständlich nicht, dass der unbekannte Meister gerade diese Werke Peruginos unmittelbar vor Augen gehabt haben misse, es handelt sich mir nur um die Feststellung der seinem umbrischen Vorbilde überhaupt geläufigen Typik, die ja keineswegs gerade auf diese wenigen besonders eklatanten Beispiele beschränkt ist. Auftallend ist ferner der Profilkopf des h. Paulus auf dem anderen Flugel mit dem eines Jüngers verwandt, der in Peruginos Schlüsselübergabe in der Sixtinischen Kapelle unmittelbar hinter Christus zu sehen ist, nur dass er auf dem Altarflügel etwas jugendlicher als dort erscheint. Starke Verwandtschaft mit den für Perugino charakteristischen Gesichtsformen zeigen auch die h. Anna in der Begegnung mit Joachim, und die klagende Frauengestalt die im Mittelbilde rechts hinter der Madonna steht, auch in den landschaftlichen Fernsichten aller Bilder wird man Einzelheiten gewahr werden, die an jenen erinnren, so in der eigentumlichen Bodenplastik des Hauptbildes und in den sehr bezeichnenden Formen der schmächtigren, dunnbelaubten Baumstämme, die durchweg im Mittelgrunde der einzelnen Tafeln wiederkehren. Es sind dieselben Elemente, mit denen auch Pinturicchio seinen Aufwand an landschafticher Dekoration zu bestreiten pflegt. Und endlich kann es uns nicht entgehen,

1) Abbildungen der genannten Gemälde Peruginos bei Knapp, Perugino(Künstlermonographien LXXXVII B. $47,20,4 \mathrm{~T}$. 
wie auch die Komposition des dominierenden Bildes, der Beweinung Christi, sich an eine Bildform anschliesst, die zwar nicht dem Kunstkreise des Perugino allein eigentümlich ist, die aber doch in diesem mit einer unläugbaren Vorliebe verwendet worden ist. Dies ist das Bild der Schmerzhaften Mutter, die sitzend den auf ihrem Schosse ruhenden Leichnam ihres Sohnes beklagt, den zwei andere heilige Personen zur Rechten und zur Linken, Nikodemus und Joseph von Arimathia unterstutzen und gewissermassen in der Schwebe halten, ein Motiv, dessen Elemente in höchster Prägnanz in einem bekannten, für die Gesuati bei Florenz gemalten, heute in der Akademie an gleichem Orte befindlichen Bilde entwickelt sind. Besonders nahe kommt die von dem Autor des römischen Altarwerks gewählte Anordnung der Mittelgruppe - wenn wir unseren Blick auch auf den weiteren Umkreis der von Perugino abhängigen italienischen Meister richten wollen - einem grossen Altarbilde des Raffaello Botticini von I 508, das aus der Kollegiatkirche von Empoli in die Sammlung der Uffizien gelangt ist (No. I283). Das Motiv ist allerdings nicht rein von unserem Meister dibernommen worden. Er hat damit, und zwar in etwas naiver Weise, ein zweites, für die italienische Malerei der Renaissance ebenso wichtiges Kompositionschema verschmolzen, das die Grablegung Christi zum Gegenstande hat und das einerseits in einem herrlichen Kupferstich des Andrea Mantegna (B. I.) und andrerseits in etwas späterer Zeit in dem berühmten Jugendwerk des Raphael aus dem Jahre 1507 seine vollkommenste Ausgestaltung erfahren hat. Der in dem Gemälde des Camposanto sich aus diesem Verfahren ergebende Antagonismus zweier völlig konträren Bewegungsmotive, des Davontragens bei den beiden Jungern, die die Enden des Bahrtuchs ergriffen haben und des Zurückhaltens bei der ganz in die leidvolle Stimmung des Augenblicks versunkenen Madonna ist begreiflicherweise der gedanklichen Klarheit des Komposition nicht günstig gewesen. Das aber scheint dem Autor im Eifer seines Bildungsdranges nicht zum Bewusstsein gekommen zu sein.

Im ganzen also sind es, um zu dem Ausgangspunkt dieser Betrachtungen zurückzukehren, die archaischen Formen der umbrischen Schule, welche dem nach unsrer Annahme aus der deutsch-niederländischen Spätgotik herausgewachsenen Meister als ein ihm kongeniales Element am meisten zugesagt haben, und dass er gerade mit diesen in besonders nahe Berühung kam, erklärt sich wohl auf die natürlichste Weise aus dem allgemeinen Beifall, dessen sich die Umbrer in der Periode Alexanders VI, damals als auch er nach Rom kam, zu erfreuen hatten. Allein er hat dabei nicht Halt gemacht. Er trat ja mitten in die werdende Geschmacksbewegung ein, die um die Wende des 15 zum 16 Jahrhundert von der sinnlich-naiven Naturnachbildung der vorangegangenen Zeit zu der Vollkommenheit eines höheren Formgesetzes und damit zugleich zu einer erneuten und vertieften 
Aneignung der einheitlich grossen und schwungvollen Anschauungstormen der Antike einzulenken begann. Und auch die Meister, die sich auf dieser Linie der fortschreitenden Entwicklung bewegten, haben sein Formempfinden nicht unberuhrt gelassen. So scheint im besonderen LEONARDOS Genius, wenn auch vielleicht nur durch Vermittelung der mit ihm durch verwandtes Streben oder durch direkte Nachfolge verbundenen Florentiner oder Mailänder Künstler den Maler des römischen Altarbildes gestreift zu haben. Die Zuge der sitzenden Madonna in dem Bilde der Anna Selbdritt zeigen unverkennbar das Gepräge eines von LEONARDO geschaffenen Typus weiblicher Gesichtsbildung und an dieselbe Schule erinneren Einzelheiten wie das Haarband mit seinen auf beiden Seiten des Kopfes geschlungenen Schleifen, die man mit der analogen Anordung auf dem irrthümlich einst dem LEONARDO selbst zugeschriebenen Freskobilde der Madonna mit dem Stifter im Kloster von S. Onofrio zu Rom zusammenhalten mag, oder wie die Lage und Körperbildung des Bambino, für den sich in zwei Madonnabildern der Nationalgalerie in London, der oft besprochenen, von einem Verrocchioschüler herrührenden Maria mit den zwei Engeln und einer dem Boltraffio zugeschriebenen Darstellung 1) zwei nahe verwandte Kinderakte zum Vergleich anbieten. Ich erwähne diese beiden letzten Bilder wieder mit dem Vorbehalte, dass es mir dabei nicht sowohl um den Nachweis einer tatsächlichen Entlehnung, als vielmehr um die Kennzeichnung der allgemeinen Einflusssphäre zu tun ist, unter deren $Z$ wang der Schöpfer unsrer Altargemälde steht. Auch der heroische Figurenstil des Signorelli spielt in unsren Bildern eine Rolle, vielleicht ebenfalls nicht unmittelbar, aber in einer Mischung mit lombardischen Besonderheiten, wie sie ähnlich etwa der Meister des Triptychons mit der Madonna und den Heiligen Lorenz und Sebastian in der Villa Albani ${ }^{2}$ ) zeigt. Ich verdanke diesen letzten Hinweis August Schmarsow, dem auf Grund der Photographien namentlich die Begegnung von Joachim und Anna auf dem einen Flügelbilde dazu Veranlassung gab. Auch zu dem breitschultrigen Christuskörper, der so wenig mehr dem asketischen Ideal des mittelalterlichen religiösen Empfindens entspricht, durften sich bei keinem italienischen Maler dieser Zeit näher verwandte Züge als bei dem Meister von Cortona finden.

Die offenbar sehr stark entwickelte suggestive Beanlagung, die den Urheber des Flúgelaltares im Camposanto zu einem Eklektiker von so ungewöhnlicher Vielseitigkeit stempelte, hat es späteren Betrachtern in der Tat nicht leicht gemacht, die Züge seiner ursprünglichen Eigenart und damit seine wahre Herkunft

1) Abbildungen von beiden Gemälden bei v. Seidlitz, LEONARDO DA VINCI I. S. 57, II. S, I47.

2) Anderson 4500 . 
zu erkennen. Die Romfuhrer vom 16 Jahrhundert an bis auf die neueste Zeit nehmen ihn denn auch unbesehen für einen Italiener. Sie geben ihm verschiedene Namen, am häufigsten den des POLIDORO DA CARAvaGgio, den man in Rom von altersher da anzuwenden liebte, wo man einen anderen Rat nicht wusste. Nur einer von den einheimischen Kennern, dieser jedoch einer von den kundigsten, GIULIo Cesare MANCINI, der seine Nachrichten über römische Künstier und Kunstwerke in der Zeit der Pontifikate Pauls V und Urbans VIII also in den ersten Jahrzehnten des i 7 Jahrhunderts niederschrieb, nennt ihn einen Deutschen, so in einem Führer durch die römischen Gemäldeschätze, der in einer Sammlung seiner hinterlassenen Schriften in der Vatikanischen Bibliothek enthalten ist, und wo der Kirche des Camposanto mit den Worten gedacht wird: pdoue l'altar maggiore è d'un todesco, coetano di Raffaello." ") Woher MANCiNi in diesem Falle seine Informationen hatte darüber vermögen wir allerdings nur Vermutungen aufzustellen. Er kann selbständig den Versuch gemacht haben, die Persönlichkeit des fremden Meisters zu bestimmen, ebensowohl kann ihm aber auch eine mündliche Überlieferung an Ort und Stelle mitgeteilt worden sein. Wenn er den Künstler einen Zeitgenossen Raphaels nennt so wird man das im übrigen nicht so zu verstehen haben, als ob er ihn damit auch als dessen Nachahmer habe bezeichnen wollen, man kann diese Äusserung ebensowohl auf die verwandte umbrisch-florentinische Schulbildung beziehen, die beide Meister etwa gleichzeitig, wenn auch mit sehr verschieden geartetem Erfolg durchlaufen haben.

Aber was auch die Unterlagen dieser Nachricht des MANCINI gewesen sein mögen, in jedem Falle sind es der inneren und äusseren Merkmale genug, die auch

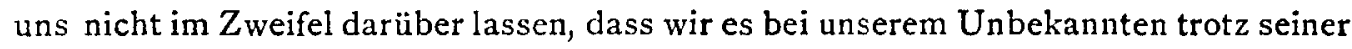
offenbaren Annäherungsversuche an die italienische Kunstweise dennoch mit einem Meister nordischer und höchst wahrscheinlich niederländischer Abkunft zu tun haben. Ist schon an sich die dem italienischen Kunstgebrauch nach I 500 gänzlich ungewohnte Gestalt des deutsch-niederländischen Flügelaltares ein Kennzeichen, das deutlich genug für sich selbst spricht, so weisen nach derselben Richtung auch gewisse Besonderheiten der Formgebung, die innerhalb der italienischen Schulgewohnheiten an verschiedenen Stelien zutage treten, mit besonderem Nachdruck aber muss dafür das Farbenmaterial des Kunstlers und seine Behandlung geltend gemacht werden. Es ist nicht mehr als natürlich, dass gerade dieses Element der malerischen Darstellung in dem gegebenen Falle die stärkste Beweiskraft in sich trägt. Der Farbensinn ist $z \mathrm{u}$ eng mit der ursprunglichen Orga-

1) Manuskript der Vatikanischen Bibliothek (Cod. Barb. Lat. 4315). Viaggio per Roma, per uedere le pitture, che in essa si ritrouano, fol. I $\mathrm{v}$. 
nisation des kunstlerischen Talents verwachsen, und die Unterschiede des koloristischen Aufnahmevermögens sind ein so durchaus integrierender Bestandteil auch in den Abweichungen, welche die nationalen Charaktere der verschiedenen historischen Schulen gegeneinander zeigen, dass selbst so weitgehende Assimilierungsvorgänge, wie sie oft genug in der Malerei der Renaissance-epoche zwischen Norden und Süden stattgefunden haben, doch so gut wie nie diese grundlegenden $Z$ üge sei es auf der einen. sei es auf der anderen Seite auszulöschen vermocht haben.

Ein Gleiches ist auch hier der Fall. Alles Bunte, was der Farbengebung der ober- und mitte-italienischen Schulen im 15 und im Beginn des I 6 Jahrhunderts als eine nur selten fehlende Eigentümlichkeit anhaftet, ist gänzlich gemieden, ja es ist im Gegensatz dazu eine Harmonisierung der Lokalfarben angestrebt wie man sie zur selben Zeit höchstens im Gebiet der venezianischen Schule in gleicher Bestimmtheit finden wird, wie sie aber vor allem der niederiändischen eigentümlich ist. Es liegt im Sinne dieser nämlichen koloristischen Tendenz, wenn sich bei dem Meister der römischen Gemälde eine ganz bestimmte Vorliebe für den Gebrauch von komplementären Farben bemerkbar macht; so stehen ein helles Grün usd Krapprot zusammen in der Gewandung der Magdalena die im Mittelbilde rechts vorne kniet, und auf einem der Aussenflugel in dem Mantel der thronenden Mutter Anna, der aus einem dunkelroten Stoff mit grünem Futter angefertigt ist. Blau und Gelb vereinigen sich in der Bekleidung des Joachim auf dem Bilde der Begegnung, ferner ist dem gedämpften Kadmiungelb, das der Mantel des Jakobus zeigt, der dunkelblaue Leibrock seines Partners, des Apostels Paulus gegenubergestellt, und dieselbe Farbenverbindung wiederholt sich ein drittes $\mathrm{Mal}$ in der händeringenden Frauengestalt ganz rechts im Hauptbilde. Überhaupt ist Gelb als Gewandfarbe besonders oft verwendet, ebenso ein starkes, ziemlich dunkles Zinnoberrot, das beispielsweise auf den Flügeln der Mantel des Paulus, und in noch tieferer Tönung der von Anna in der Begegnung und der von Johannes zeigt, endlich ebenso der Kleidrock Marias in der Anna Selbdritt und wieder wie schon erwähnt, auf dem gleichen Bilde der Mantel Annas. Im Hauptbilde tritt das Rot etwas zurück zugunsten des Ultramarinblau, das die Muttergottes auszeichnet, und einiger bunteren Farben, zu welch letzteren ein kräftiges Stahlblau und ein tiefes Olivgrün gehören, das Rock und Mantel des Trägers auf der linken Seite zeigen, sowie ein feines Aschgrau, das der Kleidrock der in der Mitte stehenden klagenden Frau und der Mantel des Trägers rechts aufweisen.

Ich glaube, diese Aufzählung allein wird genügen, um fühlbar zu machen, wie weit sich das Kolorit unsrer Tafel von der gemeinen italienischen Kunstweise entfernt, wăhrend es sich ebenso entschieden den Gewohnheiten der altnieder- 
ländischen und namentlich der altholländischen Schule anschliesst: ENGELBRECHTSEN, JAKob CORNelisz., SCOREL in seinem fruhesten Obervellacher Werk, das sind die zunächst verwandten Typen farbiger Komposition, welche die römischen Bilder in unserer Erinnerung wachrufen. Und diese Eindrucke machen sich uns noch stärker fühlbar vermöge des ungemein edlen, tiefen und doch leuchtenden Gesamttons, in dem es dem Maler geglückt ist, sein Bild zusammenzuhalten: der schönste Perugino erscheint grau und stumpt gegenüber der warmen und starken Brillanz, mit der er seine Farben in Wirkung gesetzt hat.

Sind somit in der Bedingtheit der koloristischen Haltung die sichersten Merkmale gegeben, um nordische und sudliche Eigenart scharf auseinderzuhalten, und berechtigen sie uns auch in der schwebenden Frage, mit Bestimmtheit für die niederländische Abkunft unseres Meisters einzutreten, so sind dies doch nicht die einzigen Kriterien, die uns zur Verfügung stehen. Vielmehr lassen sich ebenso wie schon bemerkt, hinsichtlich der reinen formalen Anschauung gewisse Erscheinungen namhaft machen, nach denen sich die nationalen Unterschiede mit Deutlichkeit gegeneinander abgränzen. Es überwiegt selbst bei den uberzeugtesten Romanisten in der niederländischen Renaissanceperiode des 16 Jahrhunderts ein naturalistisches Grundgefühl, das sie scheidet von der italienischen Art der Formbezeichnung. Ein MABUSE, ein SCOREL, ein HEEMSKERK, um nur diese klassischen Zeugen zu nennen, hängen viel zu eng mit der von der "Andacht zu den kleinen Dingen" ausgegangenen altniederländischen Überlieferung zusammen, als dass ihnen eine mehr als oberflächliche Annäherung an die Musterbeispiele italienischer Formgebung gelungen wäre. Ihr Formgefühl, das wie ein feines Messinstrument auf jedes leiseste Zittern der naturgegebenen individuellen Bildung reagiert, ist gänzlich verschieden von der spezifisch italienischen Auffassungsweise, die schon bei den Meistern des zu Ende gehenden Quattrocento und in noch weit höherem Masse bei denen der späteren Blütezeit das Auge auf die grosse Form allein gerichtet hält, die nur die Richtungslinien des im ganzen entscheidenden Bewegungsausdrucks gelten lässt. In den Rahmen einer auf solcher Grundlage erwachsenen Allgemeingestaltung fügen jene Niederländer das Kunstwerk ihrer miniaturhaften Einzelausführung hinein, aber ohne dass ein innerer Kontakt zu Stande käme zwischen dem Feingehalt der Fullung und dem umsoviel grösser gedachten Zuschnitt der umrahmenden Teile.

Diesen Gegensatz zwischen Kernform und äusserer Umhüllung, wenn wir $\mathrm{i}^{\text {hn }}$ so bezeichnen dürfen, lässt auch der Meister vom Campo Santo deutlich erkennen, am meisten natürlich da, wo er sein Können mit ganzer Kraft einsetzt, wie beispielsweise in den Figuren der Haupthandlung des Mittelbilds.

Die Art von erschöpfender Détailbehandlung, wie man sie an dem augen- 
scheinlich nach dem Modell gearbeiteten Christuskörper oder an den muskulösen Armen der Träger gewahr wird, geht weit über das hinaus, was dem italienischen Durchschnittsempfinden dieser Zeit an Akribie der Einzelausführung Bedurfnis war• Aber auch die physiognomische Charakterzeichung weist in eben derselben Gruppe von Figuren, zu welcher diese beiden gehören Züge einer rückhaltlosen Wirklichkeitsdarstellung auf, die dem romanischen Kunstgeist in dieser Epoche durchaus fremd sind. Der Träger zu Häupten des Christus mag immerhin mit seinem interessanten Profil in der Reihe der Apostelköpfe von LEONARdos Abendmahl den einen oder anderen Dop. pel gänger finden, der Gesichtstypus der Magdalena, die den herabhängenden Fuss des Todten mit ihren Händen umfasst mag mit ihrer breiten Stirn und den regelmässigen Formen der gesamten Kopfbildung an bekannte Heiligenbilder Signorellis erinnern: der heftige Ausdruck seelischer Schmerzempfindung, den die in tiefe Falten gelegten Mienen beider Gestalten zeigen, steht in einem unverkennbaren Gegensatz zu der Masshaltung, die wir in diesen selben $Z$ ügen da, wo sie uns nicht in abgeleiteter Anwendung, sondern in der Urform gegenubertreten, zu finden gewohnt sind. Vollends die vom Weinen verzerrten Physiognomien der Madonna und des robusten Trägers, der am Fusz-ende des Bahrtuches zugreift, verraten, obwohl sie tief und wahr empfunden sind, doch eine bis auf die Spitze getriebene Betonung des Affektes, die in einem deutschen oder niederländischen Kunstwerk dieser Zeit nicht befremdlich wirkt, die aber nichts von jenem gehaltenen von ächt antikem Geist erfüllten Pathosstil erkennen lässt, den wir in analogen Darstellungen der gleichzeitigen italienischen Schule, bleiben wir etwa gerade bei dem Beispiel Peruginos oder Signorellis, nie vermissen werden. So wird das Formgerüst, das unser Meister von seinen italienischen Kunstgenossen entlehnt hat doch an wesentlichen und sogar entscheidenden Punkten, ihm selbst vielleicht völlig unbewusst, doch wieder verleugnet durch die ursprünglichen Kennzeichen seiner heimatlichen Art.

Wir haben endlich noch eines äusseren Umstandes zu gedenken, der zu Günsten des von uns versuchten Nachweises spricht und der in der Tatsache gegeben ist, dass allgemein die beiden deutschen Nationalkirchen der Anima wie des Campo Santo den stammverwandten Kunstlern, die nach Rom kamen, oft und gerne lohnende Arbeitsgelegenheit gegeben haben. Den Spuren dieser bevorzugten Stellung, die den Landsleuten ober- und niederländischer Zunge in beiden Gemeinden eingeräumt war, begegnen wir in ihren Kirchen noch heute. Noch schmücken das Innere van S. Maria dell' Anima die Wandgemälde von der Hand des Michiel van Coxie und eine Reihe stattlicher Grabdenkmäler, die Werke des Nikolaus von Arras, des Egidio Fiammingo und des Duquesnoy.

Und noch zahlreicher sind die Überreste wie die Überlieferungen von deutsch-nieder]ändischer Kunsttätigkeit in der Kirche des Campo Santo. Hier 
finden wir schon aus Hadrians VI Zeit in einer neben dem Chor an dessen Evangelienseite gelegenen Kapelle, in der seit 15I7 die Schweizergarde einen Altar und ein Begräbnis hatte, die Reste eines Freskenzyklus mit Szenen der Passion, der höchst wahrscheinlich einem zugewanderten oberdeutschen, wenn auch vielleicht in Rom gebildeten Künstler zuzuschreiben ist. Sicher deutschen Ursprungs ist an gleichem Orte eine grosse Altartafel mit der Kreuzigung Christi, deren Autor dem Hans Baldung Grün nicht fernsteht, und die jedenfalls von einem der Altäre der Kirche stammt, 1) und nicht minder hat man sich im 17 Jahrhundert der kunstgeübten Hand eines Niederlănders, des JAKOB DE HASE von Antwerpen bedient, um die Wände des Chores mit decorativen Panneaux ausstatten zu lassen. 2) Diese letzten Gemälde wurden zwar bei der Errichtung des neuen Hochaltares im 18 Jahrhundert wieder entfernt, noch ist aber am nordwestlichen Vierungspfeiler der mit einem reizenden Putto geschmückte marmorne Grabstein jenes de Hase selbst erhalten, wiederum die Arbeit eines niederländischen Kunstlers und vielleicht in Wirklichkeit des FRANS DUQUESNOY, dem sie herkömmlicherweise zugeschrieben wird. Von bedeutenden Malereien, die der im Jahre I600 iu Rom verstorbene Arrigo Fiammingo in Oel und in Fresco in der Kirche des Campo Santo ausgeführt hat, erfahren wir durch die Künstlerbiographien des Baglione ${ }^{3}$ ) und des Niccolò Pio *). Kurz, man erkennt aus alledem, wie häufig Bestellungen bei deutschen und niederländischen Künstlern für die deutschen Kirchen Roms stattfanden, und vermutlich sind diejenigen, von denen wir wissen, nur ein kleiner Teil derer, die in Wirklichkeit zur Ausfuhrung gelangt sind. Umsoweniger Ursache haben wir, überrascht zu sein, wenn uns die Gewissheit eines analogen Vorgangs auch in den hier besprochenen Gemälden des Hochaltares vor Augen tritt.

Ist nun aber der Meister vom Campo Santo, wie wir ihn vorläufig nennen wollen, ein Niederländer von Geburt, so gewinnt sein Auftreten in Rom im Jahre I502 eine geschichtliche Bedeutung, die über den zunächst liegenden örtlichen

1) Das Werk gehörte vermutlich zu einem der acht im Jahre ${ }_{5} 17$ errichteten Nebenaltäre. Ein altare S. Crucis wird in einem Altarverzeichnis von 1536 genannt (Arch. Campi Sancti. Liber primus scripturarum Archiconfraternitatis Beatae Mariae Campi Sancti, fol. 227 v.). Das merkwürdige psychologische Problem, das die in manchen Dingen so ganz skrupellose unterwerfung der niederländischen Romanisten unter die italienische Schablone dem modernen Empfinden immer wieder zu raten aufgiebt - man vergleiche die treffenden Bemerkungen van CarL Justi pelegentlich Scorels im Jahrbuch der Preussischen Kunstsammlungen 11, I88 r, S. 200 - findet hier eine Parallele in der Schöpfung eines oberdeutschen Meisters derselben Zeit. Seine bodenwüchsige Eigenart hat er zwar in seiner Arbeit stärker als der Meister des Hochaltares bewahrt, er hat aber doch kein Bedenken getragen, das altbekannte Motiv der unter und neben dem Kruzifixus schwebenden Engelsgestalten in Formen zu kleiden, die dem landläufigen typus forentinischer und umbrischer Kreuzigungsdarstellungen des $I_{5}$ Jahrhunderts fast buchstäblich entlebnt sind.

2) DE WAAL a. a. O. S. I53, I5.

3) Giovanni Baglione, le vite de' pittori etc. $(1642)$ S. 78.

1) J. A. F. Orbaan, Bescheiden in Italie, eerste deel, Rome (IgII) S. 258.

Oud-Holland, I9I 3 
Interessenkreis nicht unerheblich hinausreicht. Unter den Niederländern, die es sich angelegen sein liessen, dem italienischen Vorbild nachzueifern, und zwar nicht nur in dieser oder jener praktischen Nutzanwendung, sondern in der völligen Hingabe ihres Selbst an den Gedanken eines neuen und ihnen, wenigstens nach ihrem Dafurthalten, überlegenen Bildungsideals, unter diesen Vollblut-Romanisten ist der Meister vom Campo Santo der erste und älteste, den die Geschichte kennt. Er ist zugleich in seinem Verständnis für den monumentalen Formcharakter der italienischen Kunst neben SCOREL wohl das empfänglichste unter allen jenen dem Norden entstammten Talenten, die sich der zaubergleichen Anziehungskraft der südlichen Kunst gefangen gaben. Und wenn jener sich in den seiner Obhut unterstellten Räumen des Vatikans zum bekenntniseifrigen Anhänger Raphaels und Michelangelos umgewandelt hat, so ist unser Meister, der gerade zwanzig Jahre vor ihm in Rom erscheint, der ebenso gelehrige Schüler der diesen beiden vorangehenden alteren Kunstlergeneration geworden.

Als JULiUs II in den Jahren 1507-08 zuerst die Ausmalung des vatikanischen Palastes in Angriff nahm, war, wie uns Muntz berichtet, neben den dazu berufenen italienischen Meistern, einem Sodoma, Perugino, Pinturicchio, Signorelli, Bramantino, Lorenzo Lotto, Spagna, auch ein Niederländer, JolranN Ruysch mit Namen beschäftigt. ${ }^{1}$ ) Wollten wir uns ein Bild von dem Können eines niederländischen Malers entwerfen, der wie dieser RUYSCH würdig erscheinen konnte, Seite an Seite mit den anerkannten Grössen der einheimischen Schule dem päpstlichen Kunsthaushalte eingereiht zu werden, wir könnten dafür kein passenderes Beispiel finden, als das des unbekannten Meisters, der im Jahre 1502 den Altarschrein für den Campo Santo der Deutschen geschaffen hat. Allein wir wollen uns nicht in eine Ideenassoziation verlieren, deren weitere Verfolgung in diesem Augenblick zum mindesten verfrüht sein dürfte, und so mag es bei dem Gesagten sein Bewenden haben. Mein $Z$ weck ist erreicht, wenn sich diejenigen Forscher, die sich das Studium der altniederländischen Kunst zur besonderen Aufgabe gemacht haben, durch diese Zeilen zu weiteren Nachforschungen angeregt fühlen sollten. Zu welchem Ende aber diese auch führen mögen, soviel ist gewiss, dass der Altar von S. Maria di Campo Santo in Zukunft einen nicht zu ubersehenden Markstein in der Geschichte jener denkwürdigen Epoche der niederländischen Kunst bedeuten wird, die der Renaissance im Norden zum Siege verhalf.

1) Müntx, Raphael S. 3×9. 